\title{
Long noncoding RNA DLEU2 drives the malignant behaviors of thyroid cancer through mediating the miR-205-5p/TNFAIP8 axis
}

\author{
Jiwen Yang ${ }^{1}$, Yayin Huang ${ }^{2}$, Bohan Dong ${ }^{3}$ and Yunhai Dai ${ }^{1}$ \\ 'Department of Nuclear Medicine, Yijishan Hospital of Wannan Medical College, Wuhu City, Anhui Province, China \\ 2Department of Clinical Laboratory, The Second People's Hospital of Wuhu, Wuhu City, Anhui Province, China \\ ${ }^{3}$ Department of Biochemistry and Molecular Biology, Wannan Medical College, Wuhu City, Anhui Province, China
}

Correspondence should be addressed to Y Dai: kmzzzj@163.com

\begin{abstract}
Objective: Considering the plight in thyroid cancer therapy, we aimed to find novel therapeutic targets from a molecular perspective.

Methods: Quantitative real-time PCR (qRT-PCR) and Western blot assay were carried out to determine RNA and protein expression. Cell counting kit-8 (CCK8) assay, flow cytometry, transwell migration assay and aerobic glycolysis analysis were performed to analyze cell proliferation, apoptosis, migration and aerobic glycolysis of thyroid cancer cells. MiRcode and Starbase software were used to search the downstream genes of long noncoding RNA (InCRNA) deleted in lymphocytic leukemia 2 (DLEU2) and microRNA-205-5p (miR-205-5p), and the intermolecular combination was confirmed by dual-luciferase reporter assay. The in vivo role of DLEU2 in tumor growth was verified using the murine xenograft model.

Results: DLEU2 and tumor necrosis factor- $\alpha$-induced protein 8 (TNFAIP8) were highly expressed in thyroid cancer tissues and cell lines. DLEU2 and TNRAIP8 promoted the proliferation, migration and aerobic glycolysis and restrained the apoptosis of thyroid cancer cells. DLEU2/miR-205-5p/TNFAIP8 signaling axis was identified in thyroid cancer cells. TNFAIP8 overexpression largely rescued the malignant phenotypes in DLEU2silenced thyroid cancer cells. DLEU2 positively regulated TNFAIP8 expression by acting as miR-205-5p sponge in thyroid cancer cells. DLEU2 silencing blocked the growth of xenograft tumors in vivo.

Conclusion: InCRNA DLEU2 exerted a pro-tumor role to promote proliferation, migration and aerobic glycolysis while repressing the apoptosis of thyroid cancer cells via miR205-5p/TNFAIP8 axis.
\end{abstract}

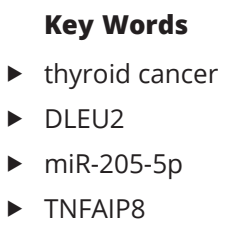

Endocrine Connections (2021) 10, 471-483

\section{Highlights:}

- IncRNA DLEU2 is significantly upregulated in thyroid cancer tissues and cell lines.

- DLEU2 accelerates the proliferation, migration and aerobic glycolysis while inhibits the apoptosis of thyroid cancer cells.
- miR-205-5p is a direct target of DLEU2 in thyroid cancer cells, and TNFAIP8 could bind to miR-205-5p in thyroid cancer cells as well.

- DLEU2 facilitates the development of thyroid cancer through upregulating TNFAIP8 via sponging miR205-5p in vivo and in vitro. https://ec.bioscientifica.com

https://doi.org/10.1530/EC-21-0046 (c) 2021 The authors Published by Bioscientifica Ltd

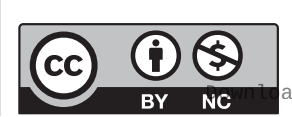

This work is licensed under a Creative Commons Attribution-NonCommercial 4.0 International License. ded from Bioscientifica.com at 04/26/2023 12:36:34PM 


\section{Introduction}

Thyroid cancer is a common endocrine malignant cancer (1). The survival of patients with thyroid cancer has improved with the combined therapy of surgery and radioactive iodine (RAI) therapy. However, the prognosis of thyroid cancer patients remains dismal due to neoplasm recurrence or other reasons. We aimed to explore the pivotal molecules involved in thyroid cancer progression.

Long noncoding RNAs (lncRNAs) are a set of RNAs that contain more than 200 nucleotides, and they are unable to code proteins. IncRNAs participate in multiple cellular functions, such as tumorigenesis and metastasis $(2,3,4)$. IncRNAs exert their functions through sponging miRNAs to suppress their biological functions $(5,6,7)$. IncRNA deleted in lymphocytic leukemia 2 (DLEU2) played an oncogenic role in multiple cancers $(8,9,10)$. For instance, Xie et al. claimed that DLEU2 accelerated the colony formation and metastasis of glioma cells through miR-186-5p/PDK3 signaling (11). DLEU2 was upregulated in thyroid cancer (12). Nevertheless, the biological significance of DLEU2 and its interaction with other factors in thyroid cancer remains poorly understood.

miRNAs are another class of noncoding RNAs. They could modulate diverse cellular processes, such as proliferation and metastasis by reducing the abundance of target messenger RNAs (mRNAs) $(13,14,15,16)$. miR-205-5p was involved in vascularization and epithelial-to-mesenchymal transition (EMT) (17). Li et al. found that miR-205-5p suppressed the metastasis of prostatic carcinoma cells via downregulating ZEB1 (18). Tao et al. claimed that miR-205-5p inhibited the growth of pulmonary vascular smooth muscle cells via MICAL2/ERK1/2 signal pathway (19). Vosgha et al. demonstrated that miR-205 restrained the progression of anaplastic thyroid carcinoma through inhibiting angiogenesis and EMT (20). This study sought to explore the role of miR-205-5p in thyroid cancer.

Tumor necrosis factor- $\alpha$-induced protein 8 (TNFAIP8) has been identified as a TNF- $\alpha$-inducible oncogenic molecule (21). Mounting researchers have reported the oncogenic role of TNFAIP8 in a series of cancers $(22,23$, 24). Duan et al. reported that TNFAIP8 was upregulated in thyroid cancer tissues compared with that in corresponding normal tissues (25). However, the binding molecules and the functional mechanism of TNFAIP8 in thyroid cancer are not fully addressed.

This study explored the target relationship between DLEU2 and miR-205-5p, as well as the target relationship between miR-205-5p and TNFAIP8 in thyroid cancer cells.
Besides, the influences of DLEU2/miR-205-5p/TNFAIP8 signaling on the proliferation, apoptosis, aerobic glycolysis and migration of thyroid cancer cells were illustrated by in vitro and in vivo experiments.

\section{Materials and methods}

\section{Patients}

Patients with thyroid cancer $(n=70)$ were recruited in Yijishan Hospital of Wannan Medical College. The abundance of DLUE2 and TNFAIP8 was examined in thyroid cancer tissues and corresponding normal tissues. This study was conducted by the agreement of Yijishan Hospital of Wannan Medical College. Informed consent was provided by patients involved in this study.

\section{Bioinformatic analysis}

The expression of DLEU2 and TNFAIP8 in the TCGA database was analyzed using two websites (http:// ualcan.path.uab.edu/index.html) and (http://gepia. cancer-pku.cn/).

\section{Cell culture}

Human thyroid normal cell line Nthy-ori 3-1 and thyroid cancer cell lines TPC-1 and BHT-101 were obtained from BeNa Culture Collection (Beijing, China). RPMI-1640 medium (Gibco) added with 10\% fetal bovine serum (FBS; Gibco) and 10\% penicillin (100 units/mL)-streptomycin $(100 \mu \mathrm{g} / \mathrm{mL})$ mixed solution was used for the cultivation of Nthy-ori 3-1 and TPC-1 cells. BHT-101 cells were cultivated with DMEM (Gibco) added with 20\% FBS (Gibco), 100 units/mL penicillin and $100 \mu \mathrm{g} / \mathrm{mL}$ streptomycin. Cells were grown in a $37^{\circ} \mathrm{C}, 5 \% \mathrm{CO}_{2}$ humidified incubator.

\section{Quantitative real-time polymerase chain reaction (qRT-PCR)}

SYBR Green Mastermix kit (Takara, Tokyo, Japan) was used for the qRT-PCR assay. The expression of DLEU2, miR-205-5p and TNFAIP8 was calculated by $2^{-\Delta \Delta C t}$ method (26). U6 or glyceraldehyde-3-phosphate dehydrogenase (GAPDH) functioned as the internal control. The following primers were used in this study: DLEU2 (forward, 5'-TCTGGAGAACAGCCTCACTTC-3'; reverse, 5'-TGCTGAGCTAAGTAGAGGTCTC-3'), miR-205-5p (forward, 5'-TCCTTCATTCCACCGGAGTCTG-3';

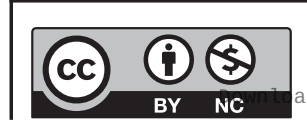

This work is licensed under a Creative Commons Attribution-NonCommercial 4.0 International License. ded from Bioscientifica.com at 04/26/2023 12:36:34PM via free access 
reverse, 5'-GCGAGCACAGAATTAATACGAC-3'), TNFAIP8 (forward, 5'-TGAAGATGGAGCACTGCTGA-3'; reverse, 5'-GGTCTGTTACCCGTTAGGAAG-3') U6 (forward, 5'-CTCGCTTCGGCAGCACATATACTA-3'; reverse, 5'-ACGAATTTGCGTGTCATCCTTGC-3'), GAPDH (forward, 5'-TGACCACAGTCCATGCCATCAC-3'; reverse, 5'-GCCTGCTTCACCACCTTCTTGA-3').

\section{Western blot assay}

Thyroid cancer cells were lysed and bicinchoninicacid kit (Beyotime, Shanghai, China) was used for protein concentration determination. The proteins were quantified, and then a total of $40 \mu \mathrm{g}$ protein samples were separated by SDS-PAGE) gel. The proteins were transferred onto a polyvinylidene fluoride (PVDF) membrane. The PVDF membrane was blocked by $5 \%$ skim milk for $1 \mathrm{~h}$ following by incubation with the following antibodies, including Ki67 (ab16667, Abcam), P53 (ab32389, Abcam), matrix metallopeptidase 9 (MMP9; ab219372, Abcam), hexokinase 2 (HK-2; ab104836, Abcam), TNFAIP8 (ab195810, Abcam) and $\beta$-actin (ab8226, Abcam). The PVDF membrane was probed with the secondary antibody (ab205718, Abcam). The protein signal was measured and quantified via the enhanced chemiluminescent (ECL) system (Beyotime) and Image J software.

\section{Cell transfection}

Transfection was performed using Lipofectamine 3000 (Invitrogen). siRNA negative control (si-NC), siRNA against DLEU2 (si-DLEU2 \#1, si-DLEU2 \#2 and si-DLEU2 \#3), siRNA against TNFAIP8 (si-TNFAIP8 \#1, si-TNFAIP8 $\# 2$ and si-TNFAIP8 \#3), shRNA negative control (sh-NC), shRNA against DLEU2 (sh-DLEU2), Vector, DLEU2 overexpression plasmid (DLEU2) and TNFAIP8 overexpression plasmid (TNFAIP8) were purchased from Genepharma (Shanghai, China). miR-NC and miR-205-5p were synthesized from Ribobio (Guangzhou, China).

\section{Cell counting kit-8 (CCK8) assay}

Thyroid cancer cells were seeded into 96-well cell culture plates at a concentration of $1 \times 10^{4}$ cells/well to assess the proliferation with Cell Counting Kit-8 (Sigma), according to the manufacturer's instructions. The absorbance at $450 \mathrm{~nm}$ was determined by a microplate reader.

\section{Cell apoptosis analysis}

The Annexin V FITC apoptosis detection kit (R\&D Systems) was used to measure the apoptosis of thyroid cancer cells. Briefly, thyroid cancer cells were stained with Annexin V combined FITC and propidine iodide (PI) simultaneously. The cells were analyzed through the flow cytometer (BD Biosciences).

\section{Transwell migration assay}

Migration assay was carried out to detect the migration ability of TPC- 1 and BHT-101 cells. TPC- 1 and BHT-101 cells were suspended in the serum-free medium and plated into the upper chambers. Medium supplemented with $10 \%$ FBS was used to fill the lower chambers. After cultivation for $48 \mathrm{~h}$, the non-migrated cells were removed with cotton swab and the migrated cells in the basal side of the membrane were stained and counted under a microscope.

\section{Aerobic glycolysis detection}

The real-time extracellular acidification rate (ECAR) was detected through an XF96 metabolic flux analyzer (Seahorse Biosciences, Billerica, MA, USA) (27). TPC-1 and BHT-101 cells were seeded into a Seahorse XF 96 cell culture microplate. $10 \mathrm{mM}$ glucose (Glc), $1 \mathrm{mM}$ oligomycin (O), and $80 \mathrm{mM}$ 2-deoxyglucose (2-DG) were injected at specific time points, respectively.

\section{Dual-luciferase reporter assay}

MiRcode online software was used for predicting the targets of IncRNA DLEU2. The wild-type or mutant type sequences of DLEU2 were inserted into the reporter vector. TPC-1 and BHT-101 cells were co-transfected with miR-NC or miR-205-5p and WT-DLEU2 or MUT-DLEU2. The Firefly and Renilla luciferase activities in TPC-1 and BHT-101 cells were detected through the Dual-GloßLuciferase Assay System (Promega). The target genes of miR-205-5p were predicted by using Starbase software. The wild-type or mutant type binding sites of TNFAIP8 3' UTR was constructed into the reporter vector. The validation of the combination between TNFAIP8 and miR-205-5p was conducted following the same approach.

\section{Murine xenograft assay}

Nude mice purchased from Beijing Vital River Laboratory (Beijing, China) were divided into two groups:

This work is licensed under a Creative Commons
Attribution-NonCommercial 4.0 International License.


sh-NC group $(n=5)$ and sh-DLEU2 group $(n=5)$. This experiment was conducted with the agreement of the Animal Research Committee of Yijishan Hospital of Wannan Medical College. TPC-1 cells were stably transfected with sh-NC or sh-DLEU2 and suspended in $150 \mu \mathrm{L}$ PBS. The mice were inoculated with the above TPC-1 cells. The dimension of tumors was recorded once a week. The mice were killed following inoculation for 4 weeks, and the tumors were excised from the mice.

\section{Statistical analysis}

All outliers have been included in our results. Data were analyzed through GraphPad Prism 7 software and presented as mean \pm S.D. Data were tested for the normality using Shapiro-Wilk test. Student's $t$-test or oneway ANOVA followed by Tukey's test was used to evaluate the differences. Spearman's correlation coefficient was utilized to analyze the linear relationship between levels of specific genes. $P$ value $<0.05$ was considered statistically significant.

\section{Results}

The abundance of DLEU2 and TNFAIP8 is upregulated in thyroid cancer

To uncover the molecular mechanism in thyroid cancer progression, we intended to explore the crucial molecules involved in thyroid cancer development. As showed in Fig. 1A, DLEU2 was conspicuously upregulated in thyroid cancer tissues compared with that in matching normal tissues. Meanwhile, the mRNA and protein expression of TNFAIP8 was also higher in thyroid cancer tissues than that in corresponding normal tissues (Fig. 1B and C). The expression of DLEU2 and TNFAIP8 in thyroid cancer in the TCGA database was also analyzed. DLEU2 expression was markedly upregulated in thyroid cancer tissues $(n=505)$ relative to normal tissues $(n=59)$ on the basis of the data in the TCGA database (Supplementary Fig. 1A, see section on supplementary materials given at the end of this article). TNFAIP8 mRNA was slightly upregulated in thyroid cancer tissues $(n=512)$ than that in normal tissues $(n=337)$. However, there was no
A
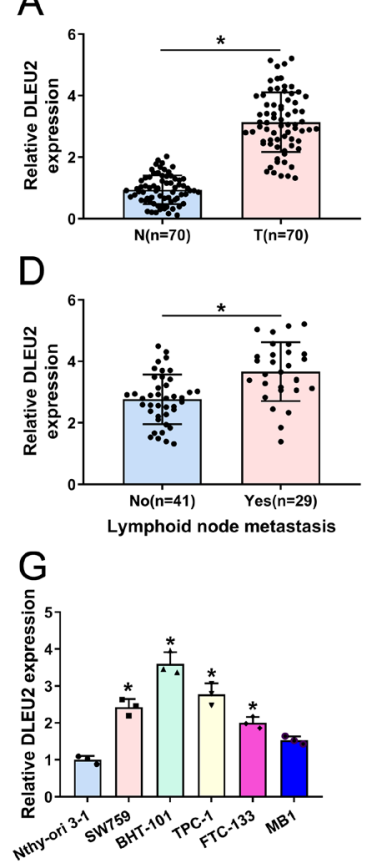
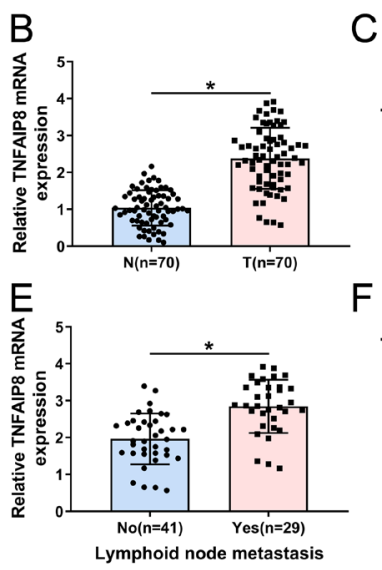

$\mathrm{H}$

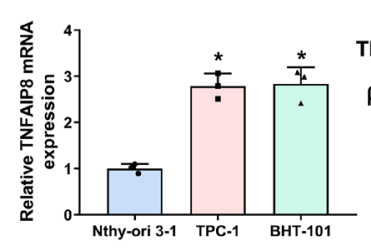

C
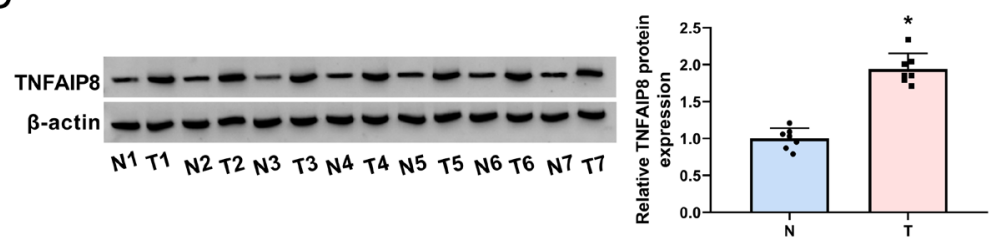

F
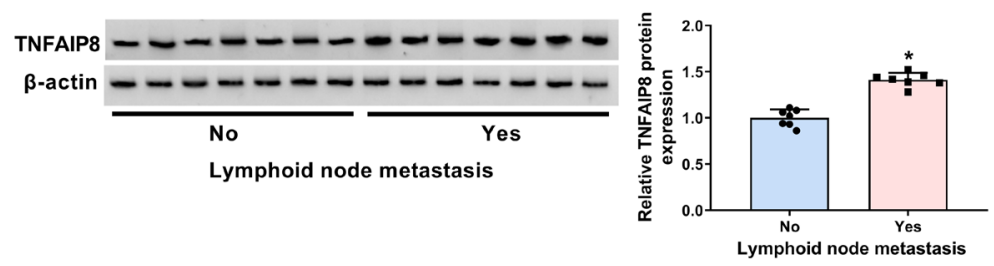

$J$

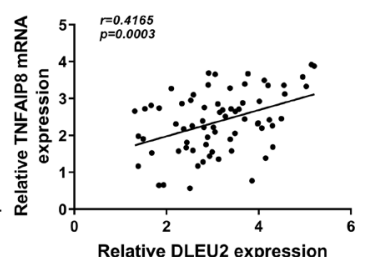

\section{Figure 1}

The abundance of DLEU2 and TNFAIP8 is upregulated in thyroid cancer. (A) qRT-PCR was applied to measure the enrichment of DLEU2 in thyroid cancer tissues and corresponding normal tissues. (B and C) The mRNA and protein expression of TNFAIP8 was determined in thyroid cancer tissues and corresponding normal tissues by qRT-PCR and Western blot assay. (D) The expression of DLEU2 was examined in lymphoid node metastatic or non-metastatic thyroid cancer tissues by qRT-PCR. (E and F) qRT-PCR and Western blot assay were conducted to detect the abundance of TNFAIP8 mRNA and protein in lymphoid node metastatic or non-metastatic thyroid cancer tissues. (G) The level of DLEU2 was measured in human thyroid normal cells Nthy-ori 3-1 and thyroid cancer cells TPC-1 and BHT-101 by qRT-PCR. (H and I) The mRNA and protein expression of TNFAIP8 was examined in human thyroid normal cells Nthy-ori 3-1 and thyroid cancer cells TPC-1 and BHT-101 by qRT-PCR and Western blot assay. (J) The correlation between DLEU2 and TNFAIP8 was analyzed in thyroid cancer tissues. * $P<0.05$.

https://ec.bioscientifica.com https://doi.org/10.1530/EC-21-0046 (c) 2021 The authors Published by Bioscientifica Ltd

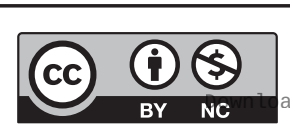

This work is licensed under a Creative Commons Attribution-NonCommercial 4.0 International License. ded from Bioscientifica.com at 04/26/2023 12:36:34PM via free access 
significant difference in the expression of TNFAIP8 in the two groups (Supplementary Fig. 1B). As indicated in Fig. $1 \mathrm{D}, \mathrm{E}$ and F, the level of DLEU2 and the abundance of TNFAIP8 mRNA and protein were higher in thyroid cancer patients with lymphoid node metastasis, suggesting that the high expression of DLEU2 and TNFAIP8 was positively related to metastasis of thyroid cancer. Apart from this, we measured the expression of DLEU2 in human thyroid normal cell line Nthy-ori 3-1 and a panel of thyroid cancer cell lines. As mentioned in Fig. 1G, DLEU2 was highly expressed in five thyroid cancer cell lines relative to Nthy-ori 3-1 cell line, especially in BHT-101 and TPC-1 cell lines. Thus TPC-1 and BHT-101 cell lines were selected for further analysis. The mRNA and protein expression of TNFAIP8 was also found to be markedly elevated in
TPC-1 and BHT-101 cell lines compared with Nthy-ori 3-1 cell line (Fig. $1 \mathrm{H}$ and I). Correlation analysis showed the expression of DLEU2 was positively correlated with the abundance of TNFAIP8 in thyroid cancer tissues (Fig. 1J). The abnormal upregulation of DLEU2 and TNFAIP8 might imply their important regulatory roles in thyroid cancer progression.

\section{DLEU2 knockdown inhibits the proliferation, migration and aerobic glycolysis while accelerates the apoptosis of thyroid cancer cells}

DLEU2 majorly located in the cytoplasmic fraction of thyroid cancer cells (Fig. 2A). To explore the function of DLEU2 in thyroid cancer cells, we conducted
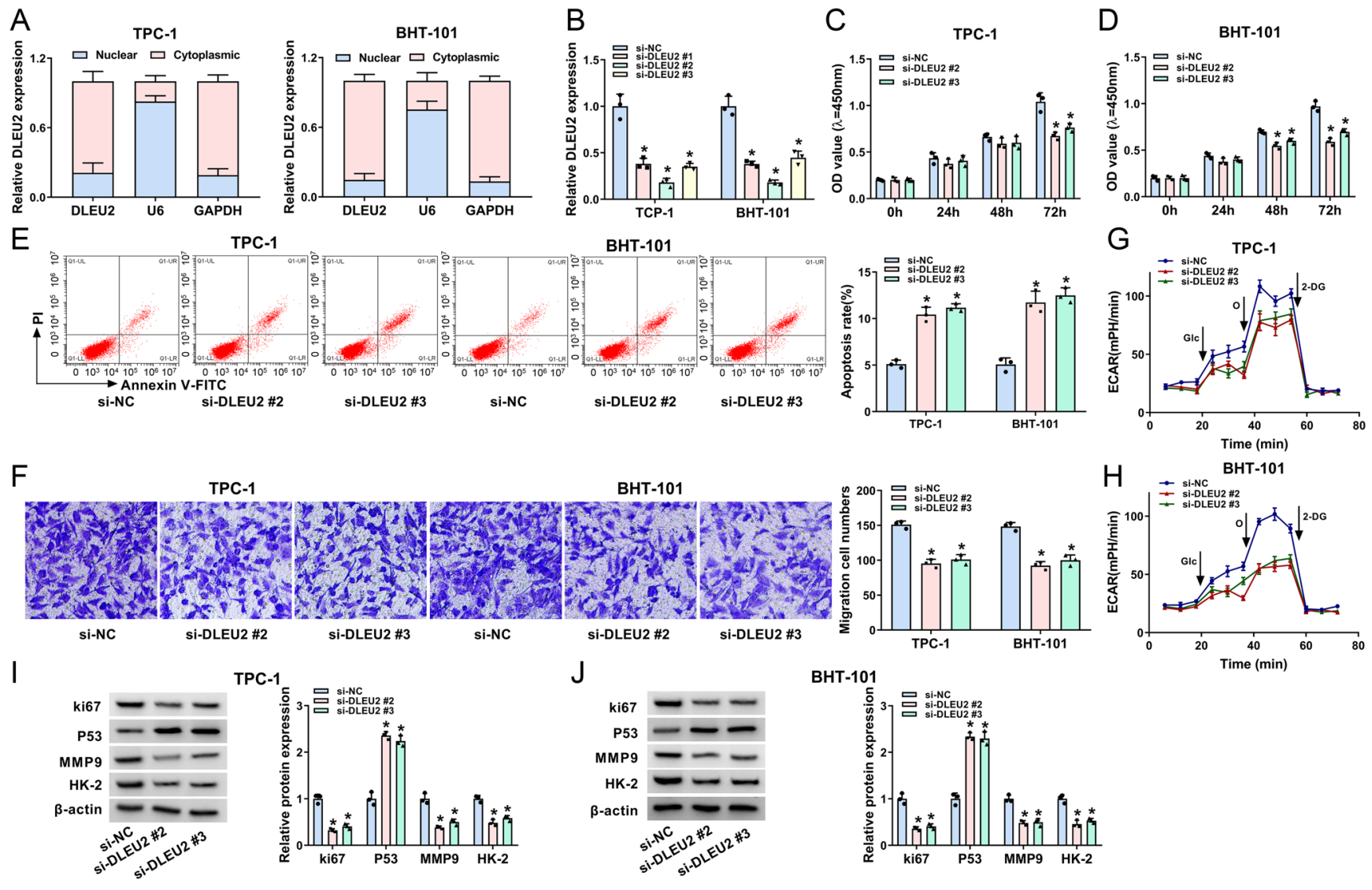

I
BHT-101

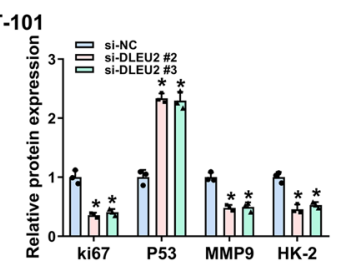

Figure 2

DLEU2 knockdown inhibits the proliferation, migration and aerobic glycolysis while accelerates the apoptosis of thyroid cancer cells. (A) The subcellular distribution of DLEU2 in the nuclear fraction and the cytoplasmic fraction of thyroid cancer cells were analyzed. (B) The enrichment of DLEU2 was determined in TPC-1, and BHT-101 cells transfected with si-NC, si-DLEU2 \#1, si-DLEU2 \#2 or si-DLEU2 \#3 by qRT-PCR. (C and D) The proliferation of si-NC, si-DLEU2 \#2 or si-DLEU2 \#3 transfected TPC-1 and BHT-101 cells weres measured by CCK8 assay. (E) Flow cytometry was performed to detect the apoptosis of TPC-1 and BHT-101 cells transfected with si-NC, si-DLEU2 \#2 or si-DLEU2 \#3. (F) Transwell migration assay was carried out to examine the migration of si-NC, si-DLEU2 \#2 or si-DLEU2 \#3 transfected thyroid cancer cells. (G and H) ECAR was analyzed in si-NC, si-DLEU2 \#2 or si-DLEU2 \#3 transfected TPC-1 and BHT-101 cells by an XF96 metabolic flux analyzer. (I and J) Western blot assay was conducted to measure the expression of proliferation-related protein (Ki67), apoptosis-associated protein (P53), migration-related protein (MMP9) and glycolysis-associated protein (HK-2) in TPC-1 and BHT-101 cells transfected with si-NC, si-DLEU2 \#2 or si-DLEU2 \#3. * $P<0.05$.

https://ec.bioscientifica.com https://doi.org/10.1530/EC-21-0046 (c) 2021 The authors Published by Bioscientifica Ltd

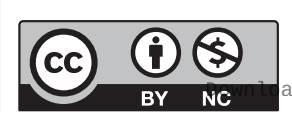

This work is licensed under a Creative Commons Attribution-NonCommercial 4.0 International License. ded from Bioscientifica.com at 04/26/2023 12:36:34PM 
loss-of-function experiments. The level of DLEU2 was notably declined in si-DLEU2 \#1, si-DLEU2 \#2 or si-DLEU2 \#3 group compared with that in si-NC group in TPC-1 and BHT-101 cells (Fig. 2B). We selected si-DLEU2 \#2 and si-DLEU2 \#3 to conduct loss-of-function experiments. As mentioned in Fig. 2C and D, DLEU2 depletion significantly suppressed cell proliferation in thyroid cancer cells. Besides, DLEU2 intervention accelerated the apoptosis of TPC-1 and BHT-101 cells (Fig. 2E). As showed in Fig. 2F, with the silencing of DLEU2, the migration of thyroid cancer cells was markedly restrained. Cancer cells prefer aerobic glycolysis rather than oxidative phosphorylation to obtain energy, and this phenomenon is called the Warburg effect (28). As we all know, the survival of cancer cells is accelerated, while apoptosis is impeded due to the Warburg effect (29). We found that the extracellular acidification rate (ECAR) was reduced in two DLEU2-silenced groups relative to si-NC group, suggesting that DLEU2 silencing suppressed the aerobic glycolysis of thyroid cancer cells (Fig. 2G and $\mathrm{H}$ ). Additionally, the results of Western blot assay also revealed that the proliferation-related protein (Ki67), migrationassociated protein (MMP9) and glycolysis-related protein (HK-2) were downregulated with DLEU2 depletion in thyroid cancer cells (Fig. 2I and J), while the abundance of apoptosis-related protein (P53) was elevated, which further demonstrated that the knockdown of DLUE2 inhibited the proliferation, migration and aerobic glycolysis whereas promoted the apoptosis of thyroid cancer cells.

To further confirm the biological functions of DLEU2 in thyroid cancer cells, we performed gain-of-function experiments. As shown in Supplementary Fig. 2A, B, C, D and E, DLEU2 overexpression facilitated the proliferation, migration and aerobic glycolysis and suppressed the apoptosis of thyroid cancer cells. The results of loss- and gain-of-function experiments together demonstrated that DLEU2 served as an oncogene to promote thyroid cancer progression.

miR-205-5p is a direct target of DLEU2, and miR-205-5p targets TNFAIP8 in thyroid cancer cells as well

miR-205-5p and TNFAIP8 were predicted as targets of DLEU2 and miR-205-5p by miRcode and Starbase software, respectively (Fig. 3A and $\mathrm{B}$ ). To verify the combination between miR-205-5p and DLEU2 or TNFAIP8 in thyroid cancer cells, we conducted dual-luciferase reporter assay. As showed in Fig. 3C and D, TPC-1 and BHT-101 cells were transfected with miR-NC or miR-205-5p and WT-DLEU2 or MUT-DLEU2, and luciferase activity was dramatically reduced in miR-205-5p and WT-DLEU2 co-transfected group, demonstrating that miR-205-5p was a direct target of DLEU2 in thyroid cancer cells. Meanwhile, we transfected miR-NC or miR-205-5p and TNFAIP8 3' UTR-WT or TNFAIP8 3' UTR-MUT into TPC-1 and BHT-101 cells. As showed in Fig. 3E and F, the overexpression of miR-205-5p notably decreased the luciferase activity in TNFAIP8 3' UTR-WT group compared with that in TNFAIP8 3' UTR-MUT group, suggesting that TNFAIP8 could bind to miR-205-5p in thyroid cancer cells. To elucidate the regulatory relationship between miR-205-5p and DLEU2 or TNFAIP8, we performed the following experiments. miR-205-5p was negatively regulated by DLEU2 in TPC-1 and BHT-101 cells (Fig. 3G). We assessed the overexpression efficiency of miR-205-5p in TPC-1 and BHT-101 cells, and the expression of miR-205-5p was markedly enhanced by miR-205-5p transfection in TPC-1 and BHT-101 cells (Fig. $3 \mathrm{H}$ ). The enrichment of TNFAIP8 mRNA and protein was inversely modulated by miR-205-5p in thyroid cancer cells (Fig. 3I and J). Taken together, miR-205-5p was a direct target of DLEU2, and miR-205-5p also could bind to TNFAIP8 in TPC-1 and BHT-101 cells. There was a negative regulatory relationship between miR-205-5p and DLEU2 or TNFAIP8 in thyroid cancer cells.

\section{TNFAIP8 plays an oncogenic role in thyroid} cancer cells

To clarify the functions of TNFAIP8 in thyroid cancer cells, we conducted knockdown experiments. As indicated in Fig. 4A, B and C, the expression of TNFAIP8 mRNA and protein was prominently downregulated by TNFAIP8 siRNA transfection in TPC-1 and BHT-101 cells. Si-TNFAIP8 \#2 and si-TNFAIP8\#3 were selected for the following knockdown experiments. TNFAIP8 depletion impeded the proliferation, migration and aerobic glycolysis while promoted the apoptosis of thyroid cancer cells (Fig. 4D, E, F, G, H and I). Meanwhile, the results of Western blot assay supported the above conclusion. The abundance of proliferation-related protein (Ki67), migration-associated protein (MMP9) and aerobic glycolysis-related protein (HK-2) was downregulated, and the expression of apoptosis-associated protein P53 was elevated in si-TNFAIP8 \#2 group and si-TNFAIP8\#3 group than that in si-NC group (Fig. 4J). These results suggested that TNFAIP8 silencing suppressed the development of thyroid cancer in vitro.

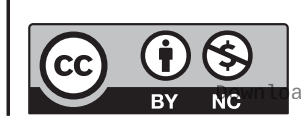

This work is licensed under a Creative Commons Attribution-NonCommercial 4.0 International License. ed from Bioscientifica.com at 04/26/2023 12:36:34PM via free access 
A

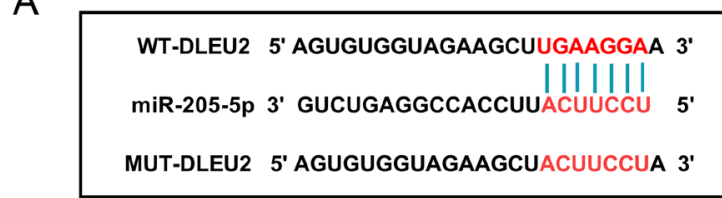

C

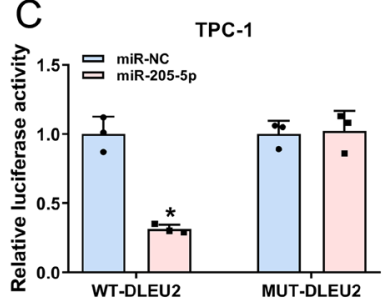

G

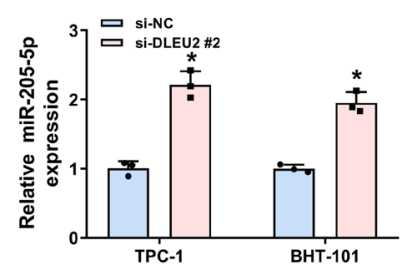

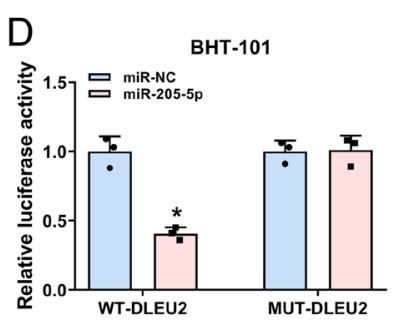

$\mathrm{H}$

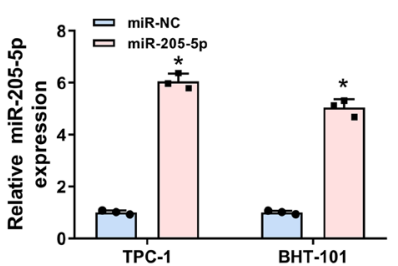

B

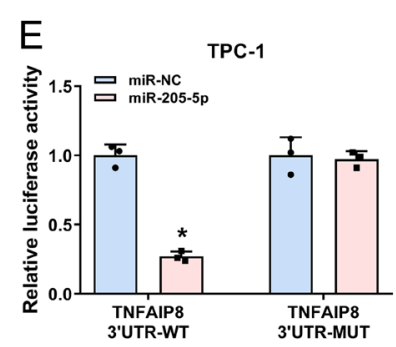

I

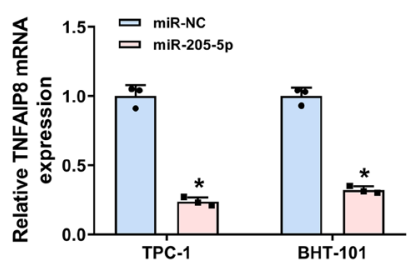

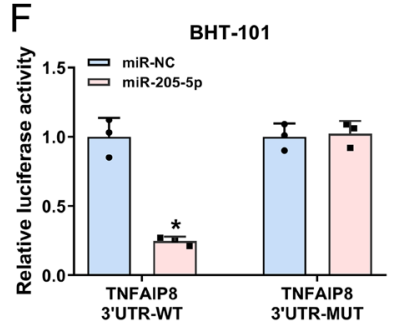

$J$

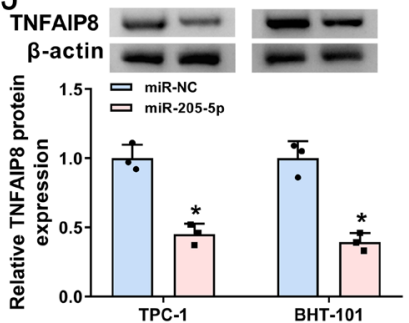

Figure 3

miR-205-5p is a direct target of DLEU2, and miR-205-5p targets TNFAIP8 in thyroid cancer cells as well. (A and B) The binding sites between miR-205-5p and DLEU2 or TNFAIP8 were predicted by miRcode or Starbase software. (C and D) Dual-luciferase reporter assay was conducted to confirm the combination between miR-205-5p and DLEU2 in TPC-1 and BHT-101 cells. (E and F) Luciferase activity was examined in TPC-1 and BHT-101 cells co-transfected with miR-NC or miR-205-5p and TNFAIP8 3' UTR-WT or TNFAIP8 3' UTR-MUT. (G) The level of miR-205-5p was detected in thyroid cancer cells transfected with si-NC or si-DLEU2 \#2 by qRT-PCR. (H) The overexpression efficiency of miR-205-5p was assessed in thyroid cancer cells transfected with miR-NC or miR-205-5p by qRT-PCR. (I and J) qRT-PCR and Western blot assay were applied to measure the abundance of TNFAIP8 mRNA and protein in TPC-1 and BHT-101 cells transfected with miR-NC or miR-205-5p. * $P<0.05$.

Gain-of-function experiments were carried out to further verify the functions of TNFAIP8 in thyroid cancer cells. TNFAIP8 overexpression facilitated the proliferation, migration and glycolysis while suppressed the apoptosis of thyroid cancer cells (Supplementary Fig. 3A, B, C, D and E). The results of loss- and gain-of-function experiments together suggested that TNFAIP8 functioned as a pro-tumor molecule to contribute to thyroid cancer development.

\section{TNFAIP8 overexpression reverses the inhibitory} effects of DLEU2 depletion on the proliferation, migration and aerobic glycolysis and the promoting impact on the apoptosis of thyroid cancer cells

The enrichment of TNFAIP8 mRNA and protein was conspicuously enhanced by TNFAIP8 transfection in thyroid cancer cells (Fig. 5A and B). TPC-1 and BHT-101 cells were transfected with si-NC, si-DLEU2 \#2, si-DLEU2 $\# 2+$ Vector or si-DLEU2 \#2+TNFRIP8, respectively. As mentioned in Fig. 5C, D and E, the mRNA and protein expression of TNFAIP8 was declined by si-DLEU2 \#2 transfection, and the addition of TNFAIP8 recovered its mRNA and protein expression in TPC-1 and BHT-101 cells. Meanwhile, we measured the proliferation, apoptosis, migration and aerobic glycolysis in the above thyroid cancer cells. The accumulation of TNFAIP8 abrogated the inhibitory effects of DLEU2 silencing on the proliferation, migration and aerobic glycolysis and the promoting impact on the apoptosis of TPC-1 and BHT-101 cells (Fig. 5F, G, H, I and J). Collectively, DLEU2 facilitated the progression of thyroid cancer cells through upregulating TNFAIP8.

\section{TNFAIP8 is modulated by DLEU2/miR-205-5p axis in thyroid cancer cells}

To clarify the relationship among DLEU2, miR-205-5p and TNFAIP8 in thyroid cancer cells, we performed the following experiments. The overexpression efficiency of DLEU2 plasmid was assessed in TPC-1 and BHT-101 cells. As showed in Fig. 6A and B, the abundance of DLEU2

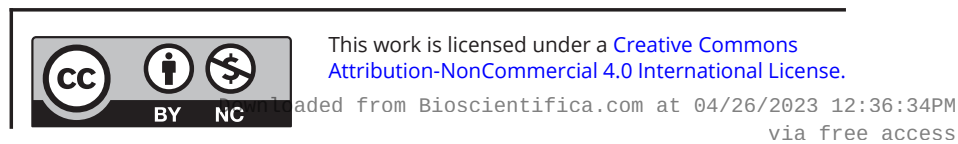


A

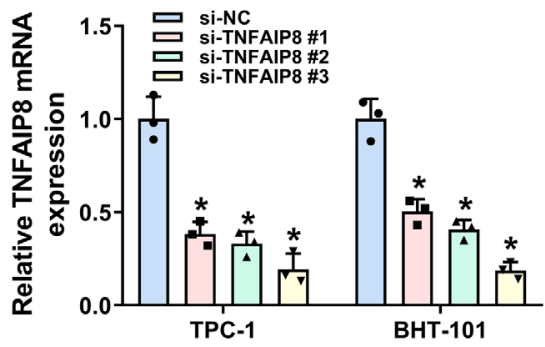

D

TPC-1

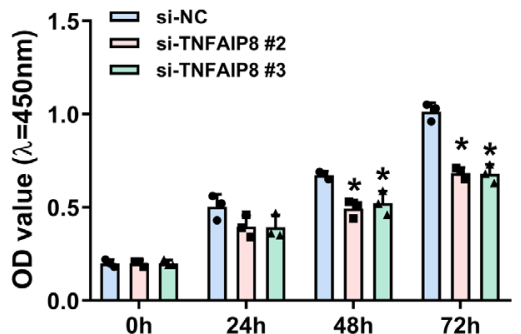

G
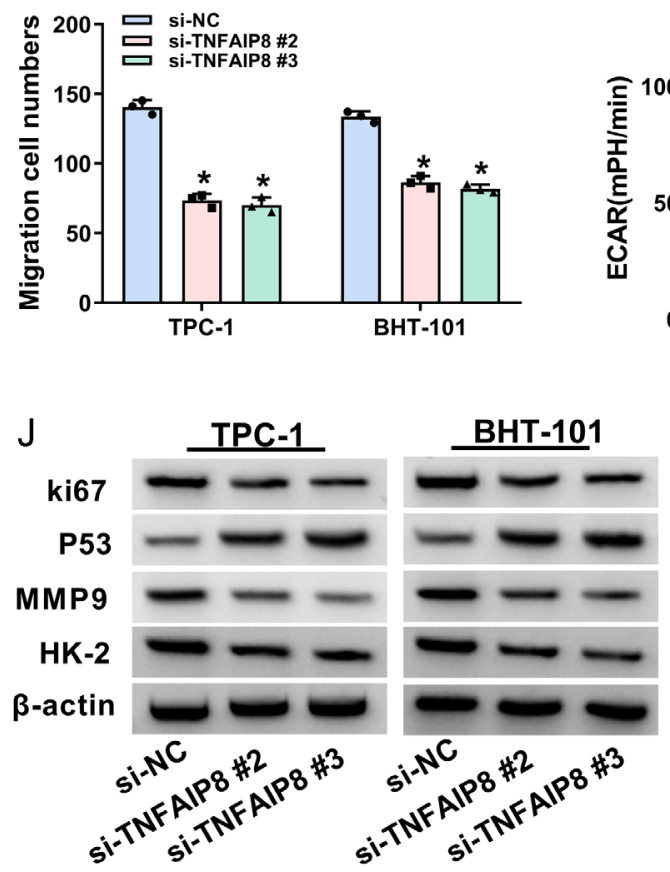

B

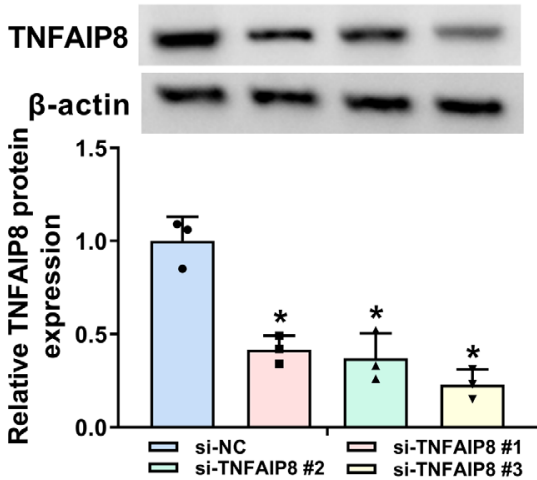

E

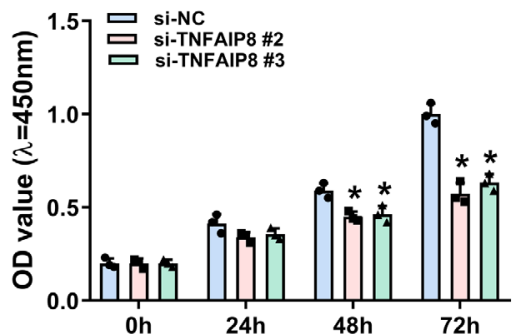

$\mathrm{H}$

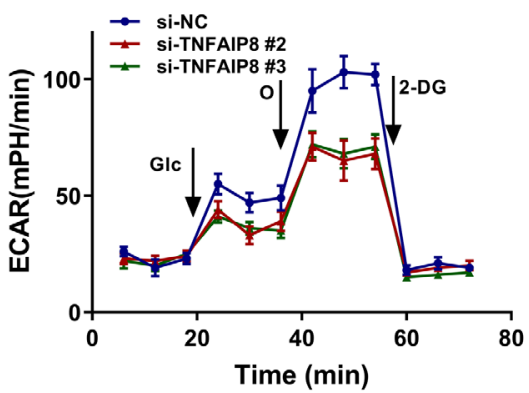

C

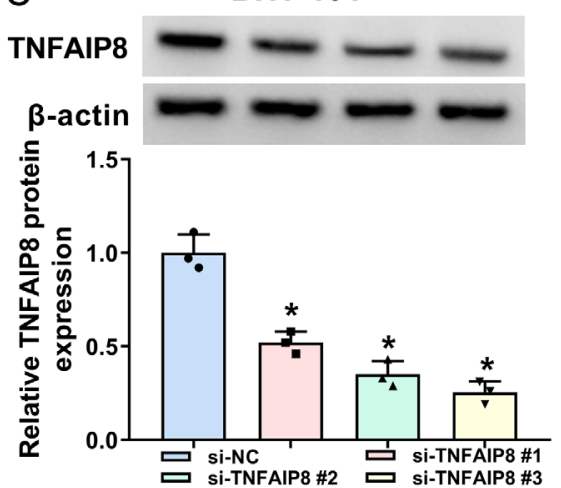

F $\square$ si-Nc

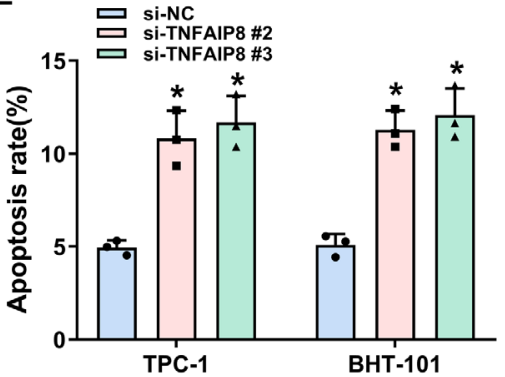

I
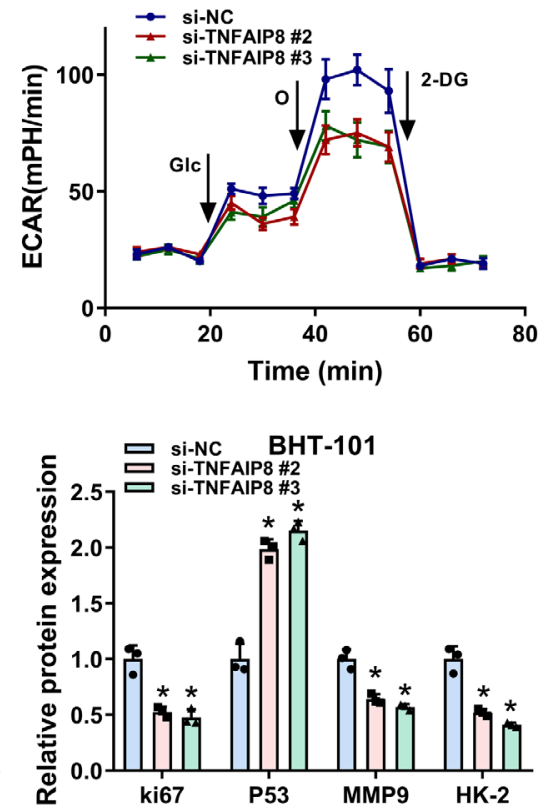

Figure 4

TNFAIP8 plays an oncogenic role in thyroid cancer cells. (A, B and C) The mRNA and protein expression of TNFAIP8 was determined in TPC-1 and BHT-101 cells transfected with si-NC, si-TNFAIP8 \#1, si-TNFAIP8 \#2 or si-TNFAIP8 \#3 by qRT-PCR and Western blot assay. (D and E) The proliferation of thyroid cancer cells transfected with si-NC, si-TNFAIP8 \#2 or si-TNFAIP8 \#3 was examined by CCK8 assay. (F) Flow cytometry was applied to detect the apoptosis of TPC-1 and BHT-101 cells transfected with si-NC, si-TNFAIP8 \#2 or si-TNFAIP8 \#3. (G) The migration of si-NC, si-TNFAIP8 \#2 or si-TNFAIP8 \#3 transfected TPC-1 and BHT-101 cells was measured by transwell migration assay. ( $\mathrm{H}$ and I) ECAR was analyzed in si-NC, si-TNFAIP8 \#2 or si-TNFAIP8 \#3 transfected TPC-1 and BHT-101 cells by an XF96 metabolic flux analyzer. () The expression of Ki67, P53, MMP9 and HK-2 in si-NC, si-TNFAIP8 \#2 or si-TNFAIP8 \#3 transfected TPC-1 and BHT-101 cells was determined by Western blot assay. * $P<0.05$.

https://ec.bioscientifica.com https://doi.org/10.1530/EC-21-0046 (c) 2021 The authors Published by Bioscientifica Ltd

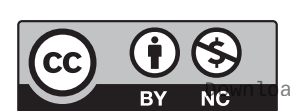

This work is licensed under a Creative Commons Attribution-NonCommercial 4.0 International License. ded from Bioscientifica.com at 04/26/2023 12:36:34PM 
A
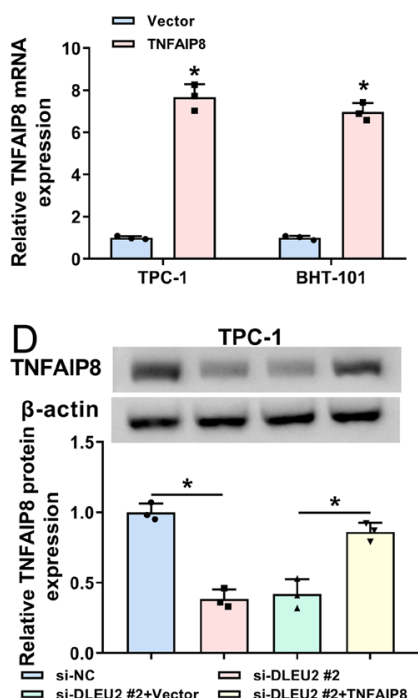

G

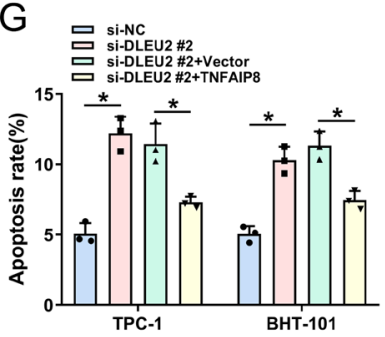

J

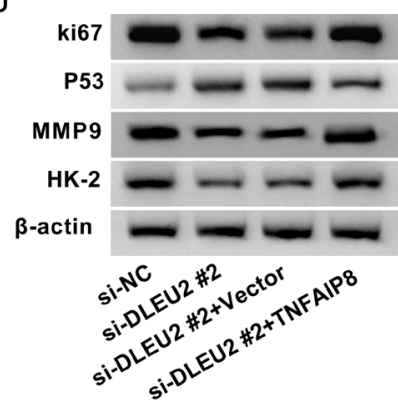

B

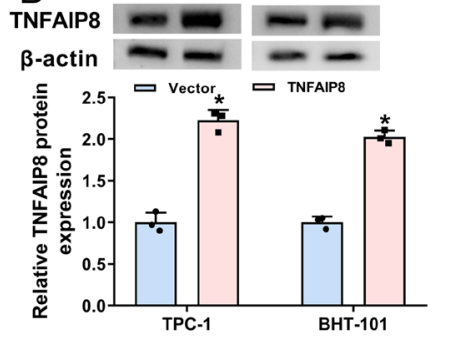

E TNFAIPB

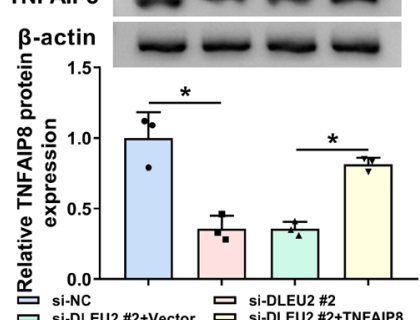

$\mathrm{H}$
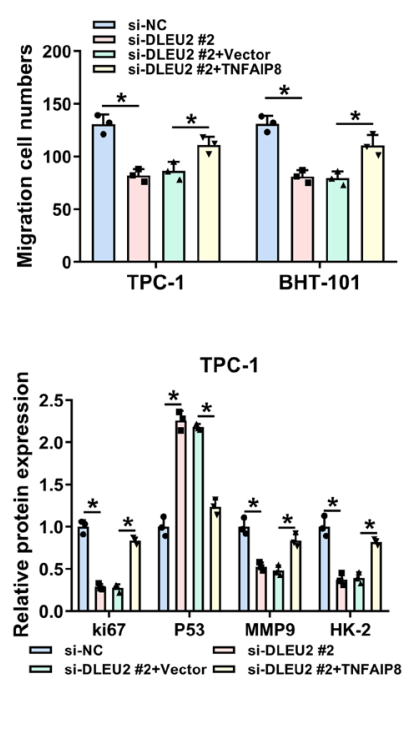

C
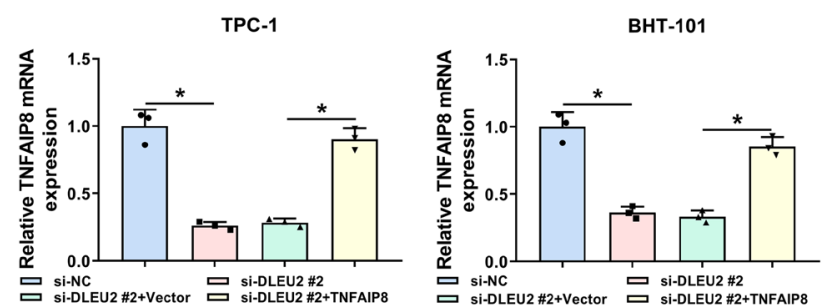

$\mathrm{F}$
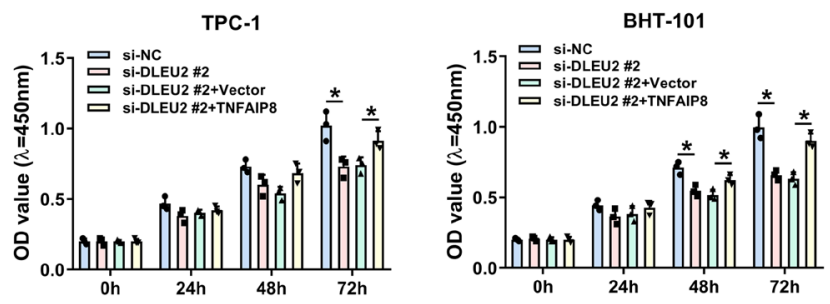

I
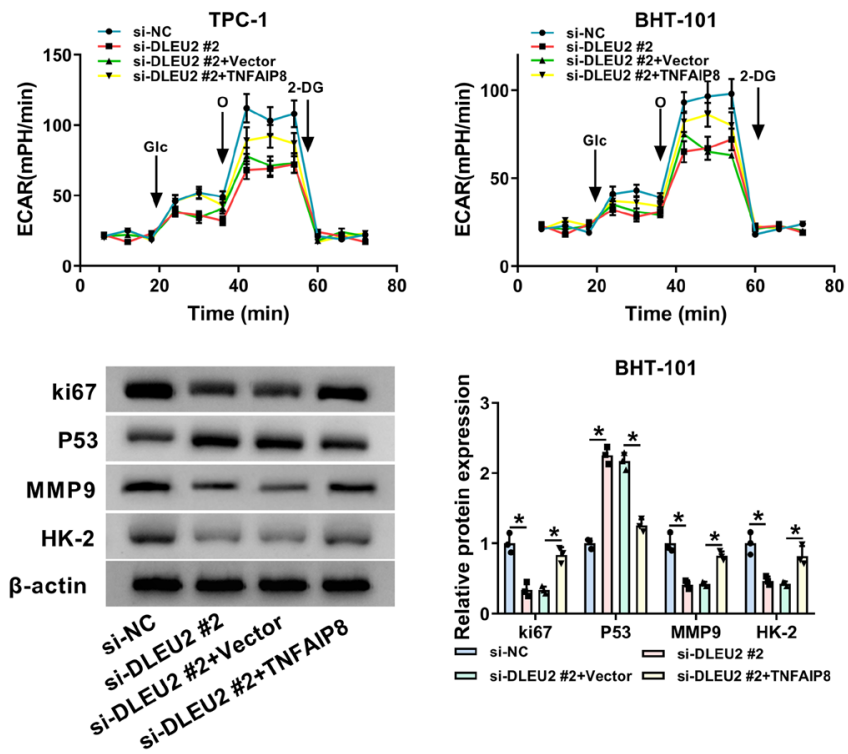

Figure 5

TNFAIP8 overexpression reverses the inhibitory effects of DLEU2 depletion on the proliferation, migration and aerobic glycolysis and the promoting impact on the apoptosis of thyroid cancer cells. (A and B) The abundance of TNFAIP8 mRNA and protein was determined in TPC-1 and BHT-101 cells transfected with Vector or TNFAIP8 by qRT-PCR and Western blot assay. TPC-1 and BHT-101 cells were transfected with si-NC, si-DLEU2 \#2, si-DLEU2 \#2 + Vector or si-DLEU2 \#2 + TNFRIP8, respectively. (C, D and E) The mRNA and protein expression of TNFAIP8 was measured in the above thyroid cancer cells by qRT-PCR and Western blot assay. (F) CCK8 assay was performed to detect the proliferation of the above TPC-1 and BHT-101 cells. (G) The apoptosis of thyroid cancer cells was examined by flow cytometry. (H) Transwell migration assay was applied to determine the migration of TPC-1 and BHT-101 cells. (I) ECAR was measured in TPC-1 and BHT-101 cells by an XF96 metabolic flux analyzer. (I) Western blot assay was performed to detect the levels of proliferation-related protein (Ki67), apoptosis-associated protein (P53), migration-related protein (MMP9) and glycolysis-associated protein (HK-2) in TPC-1 and BHT-101 cells. ${ }^{*} P<0.05$.

was conspicuously elevated in DLEU2-transfected group compared with that in Vector group in TPC-1 and BHT-101 cells. We determined the mRNA and protein expression of TNFAIP8 in TPC-1 and BHT-101 cells transfected with Vector, DLEU2, DLEU2 + miR-NC or DLEU2 + miR-205-5p.
The abundance of TNFAIP8 mRNA and protein was upregulated with DLEU2 overexpression, while the addition of miR-205-5p decreased the mRNA and protein expression of TNFAIP8 in TPC-1 and BHT-101 cells (Fig. 6C, D, E and F). Taken together, DLEU2 upregulated the

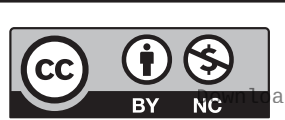

This work is licensed under a Creative Commons Attribution-NonCommercial 4.0 International License. ded from Bioscientifica.com at 04/26/2023 12:36:34PM 
A

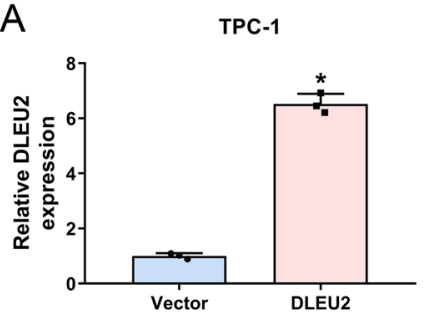

E

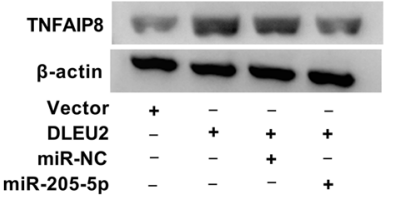

B

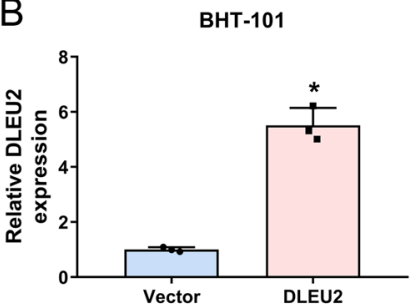

TPC-1

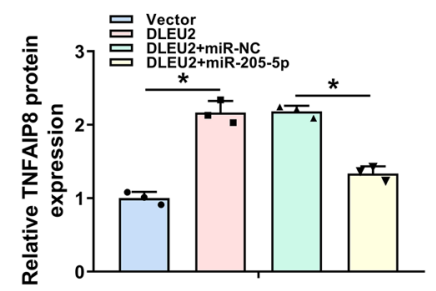

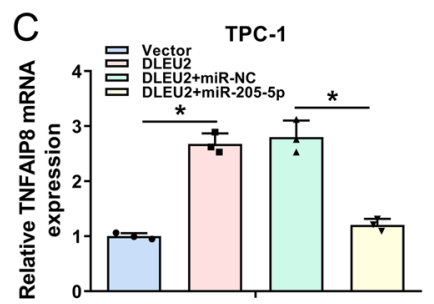

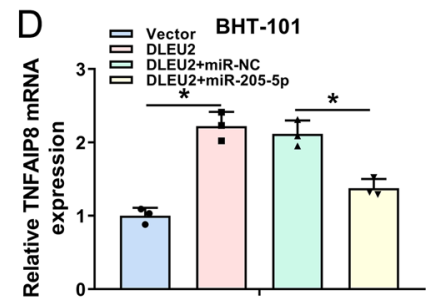

$\mathrm{F}$

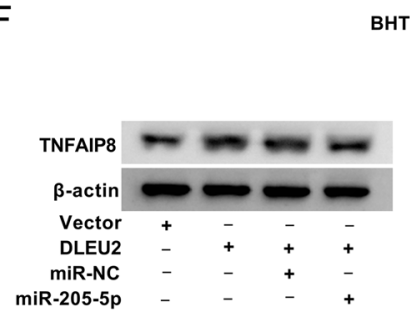

BHT-101

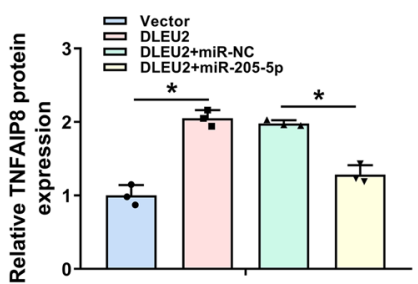

Figure 6

TNFAIP8 is modulated by DLEU2/miR-205-5p axis in thyroid cancer cells. (A and B) The expression of DLEU2 was determined in TPC-1 and BHT-101 cells transfected with Vector or DLEU2 by qRT-PCR. (C, D, E and F) The abundance of TNFAIP8 mRNA and protein was measured in TPC-1 and BHT-101 cells transfected with Vector, DLEU2, DLEU2 + miR-NC or DLEU2 + miR-205-5p by qRT-PCR and Western blot assay. * $P<0.05$.

A

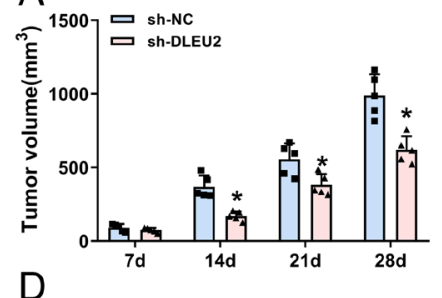

D $\quad 2 d \quad 14 d \quad 21 d \quad 28 d \quad E$

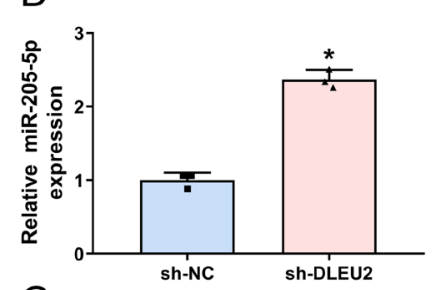

B

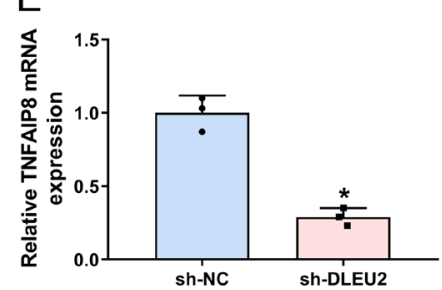

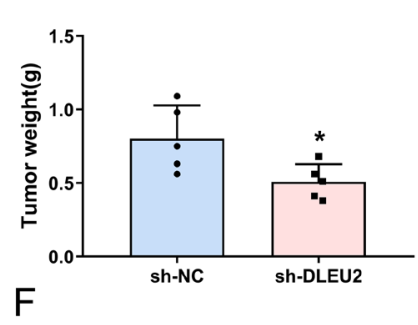
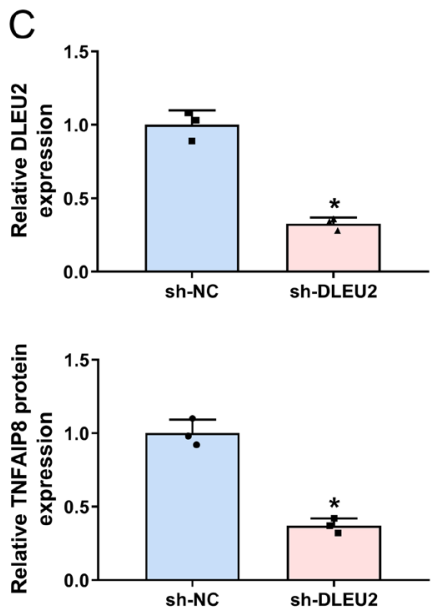

G

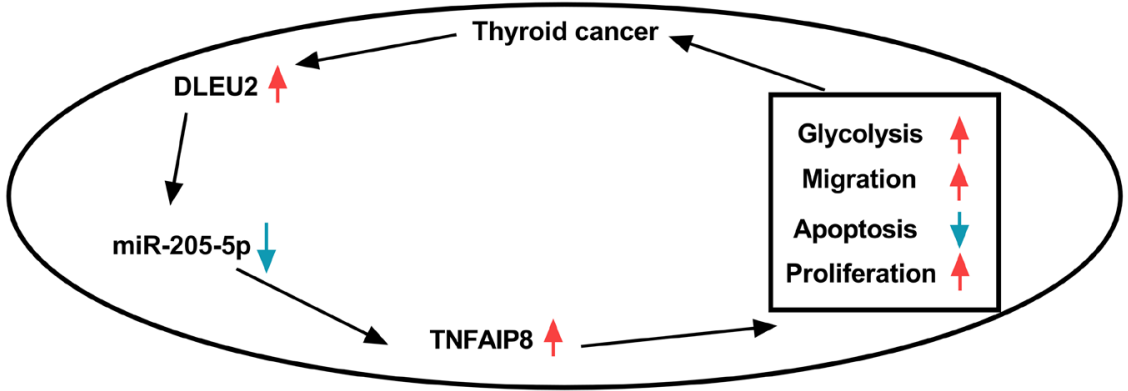

Figure 7

DLEU2 promotes the progression of thyroid cancer through miR-205-5p/TNFAIP8 axis in vivo. (A) Tumor volume was measured every 7 days. (B) Tumors were weighed after 4-week injection. (C and D) The expression of DLEU2 and miR-205-5p was detected in resected tumor tissues by qRT-PCR. (E and F) The abundance of TNFAIP8 mRNA and protein was determined in resected tumor tissues by qRT-PCR and Western blot assay. (G) A schematic illustration of our model revealed the molecular mechanism by which DLEU2 promoting the progression of thyroid cancer. $* P<0.05$.

https://ec.bioscientifica.com

https://doi.org/10.1530/EC-21-0046 (c) 2021 The authors Published by Bioscientifica Ltd

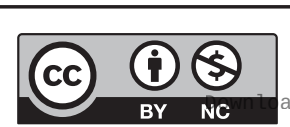

This work is licensed under a Creative Commons Attribution-NonCommercial 4.0 International License. ded from Bioscientifica.com at $04 / 26 / 2023$ 12:36:34PM 
abundance of TNFAIP8 through sponging miR-205-5p in thyroid cancer cells.

\section{DLEU2 promotes the progression of thyroid cancer through miR-205-5p/TNFAIP8 axis in vivo}

We built murine xenograft model using TPC- 1 cells stably transfected with sh-NC or sh-DLEU2 to explore the role of DLEU2 in vivo. As indicated in Fig. 7A and B, DLEU2 silencing significantly suppressed tumor growth relative to sh-NC group. The expression of DLEU2 was remarkably decreased in sh-DLEU2 group compared with that in the control group (Fig. 7C). Besides, the level of miR-205-5p was elevated while the abundance of TNFAIP8 mRNA and protein was downregulated in sh-DLEU2 group (Fig. 7D, E and $\mathrm{F}$ ). Taken together, DLEU2 promoted the progression of thyroid cancer by accelerating the proliferation, migration and aerobic glycolysis while restraining the apoptosis of thyroid cancer cells via miR-205-5p/TNFAIP8 axis (Fig. 7G).

\section{Discussion}

This study aimed to investigate the critical molecules and signal pathways involved in thyroid cancer progression. IncRNA DLEU2 was aberrantly upregulated in thyroid cancer tissues and cells compared with that in matching non-tumor tissues and thyroid normal cells Nthy-ori 3-1. DLEU2 promoted the initiation and development of multiple cancers $(30,31,32)$. However, the effect of DLEU2 in thyroid cancer is largely unknown. We found that the high expression of DLEU2 was related to the lymphoid node metastasis in patients with thyroid cancer, implying the crucial role of DLEU2 in thyroid cancer progression. Subsequently, we found DLEU2 interference inhibited the proliferation, migration and aerobic glycolysis while accelerated the apoptosis of thyroid cancer cells.

IncRNAs serve as miRNA sponges to exert their functions generally $(5,6,7)$. To elucidate the signal pathway of DLEU2 in thyroid cancer, miRcode software was used to search the downstream genes of DLEU2. miR-205-5p was predicted as a target of DLEU2, and dualluciferase reporter assay confirmed the target relationship between miR-205-5p and DLEU2 in TPC-1 and BHT-101 cells. Subsequently, TNFAIP8 was predicted as a target of miR-205-5p by Starbase software, and the combination between miR-205-5p and TNFAIP8 was verified by dualluciferase reporter assay.
TNFAIP8 has been reported to be upregulated and acted as an oncogene in thyroid cancer (25). Consistent with the above findings, we found the abundance of TNFAIP8 was conspicuously higher in thyroid cancer tissues and cells than that in adjacent non-tumor tissues and normal thyroid cells. Meanwhile, the enrichment of TNFAIP8 was positively related to lymphoid node metastasis of thyroid cancer patients. As expected, there was a positive relationship between the abundance of TNFAIP8 and DLEU2 in thyroid cancer tissues. TNFAIP8 played an oncogenic role in thyroid cancer through promoting proliferation, migration and aerobic glycolysis and impeding apoptosis of thyroid cancer cells.

The accumulation of TNFAIP8 counteracted the inhibitory effects of DLEU2 intervention on the proliferation, migration and aerobic glycolysis and the promoting impact on the apoptosis of thyroid cancer cells. Additionally, we found that DLEU2 upregulated the level of TNFAIP8 through sponging miR-205-5p in thyroid cancer cells. DLEU2 knockdown also suppressed the growth of thyroid tumors through miR-205-5p/TNFAIP8 axis in vivo.

In future, the role of DLEU2 in the invasion of thyroid cancer cells in vitro needs to analyzed. Additionally, the in vivo role of DLEU2 in the metastasis of thyroid cancer needs to be assessed in nude mice to better illustrate the biological significance of DLEU2 in the progression of thyroid cancer.

In conclusion, lncRNA DLEU2 facilitated the progression of thyroid cancer through promoting proliferation, migration and aerobic glycolysis and restraining apoptosis of thyroid cancer cells via miR-205-5p/TNFAIP8 signaling.

Supplementary materials

This is linked to the online version of the paper at https://doi.org/10.1530/ EC-21-0046.

\section{Declaration of interest}

The authors declare that there is no conflict of interest that could be perceived as prejudicing the impartiality of the research reported.

\section{Funding}

This work was supported by the Anhui University Natural Science Research Project (KJ2019A0415).

Patient consent

Written informed consent was obtained from all enrolled patients.

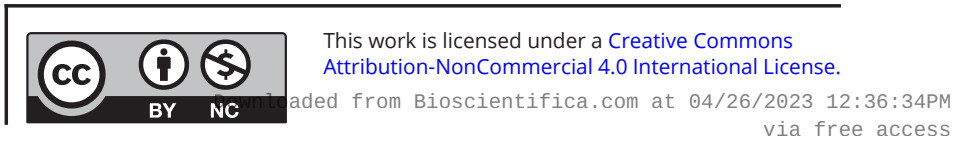




\section{Ethics approval}

The present study was approved by the ethical review committee of The Second Hospital of Hebei Medical University.

\section{Availability of data and materials}

The analyzed data sets generated during the present study are available from the corresponding author on reasonable request.

\section{Author contribution statement}

All authors made substantial contribution to conception and design, acquisition of the data, or analysis and interpretation of the data; take part in drafting the article or revising it critically for important intellectual content; gave final approval of the revision to be published; and agree to be accountable for all aspect of the work.

\section{References}

1 Kondo T, Ezzat S \& Asa SL. Pathogenetic mechanisms in thyroid follicular-cell neoplasia. Nature Reviews: Cancer 20066 292-306. (https://doi.org/10.1038/nrc1836)

2 Fan M, Li X, Jiang W, Huang Y, Li J \& Wang Z. A long non-coding RNA, PTCSC3, as a tumor suppressor and a target of miRNAs in thyroid cancer cells. Experimental and Therapeutic Medicine 20135 1143-1146. (https://doi.org/10.3892/etm.2013.933)

3 Eades G, Zhang YS, Li QL, Xia JX, Yao Y \& Zhou Q. Long non-coding RNAs in stem cells and cancer. World Journal of Clinical Oncology 2014 5 134-141. (https://doi.org/10.5306/wjco.v5.i2.134)

4 Qiu MT, Hu JW, Yin R \& Xu L. Long noncoding RNA: an emerging paradigm of cancer research. Tumour Biology 201334 613-620. (https://doi.org/10.1007/s13277-013-0658-6)

5 Cesana M, Cacchiarelli D, Legnini I, Santini T, Sthandier O, Chinappi M, Tramontano A \& Bozzoni I. A long noncoding RNA controls muscle differentiation by functioning as a competing endogenous RNA. Cell 2011147 358-369. (https://doi.org/10.1016/j. cell.2011.09.028)

6 Kallen AN, Zhou XB, Xu J, Qiao C, Ma J, Yan L, Lu L, Liu C, Yi JS, Zhang $\mathrm{H}$, et al. The imprinted H19 lncRNA antagonizes let-7 microRNAs. Molecular Cell 201352 101-112. (https://doi. org/10.1016/j.molcel.2013.08.027)

7 Wang K, Long B, Zhou LY, Liu F, Zhou QY, Liu CY, Fan YY \& Li PF. CARL IncRNA inhibits anoxia-induced mitochondrial fission and apoptosis in cardiomyocytes by impairing miR-539-dependent PHB2 downregulation. Nature Communications 20145 3596. (https://doi. org $/ 10.1038 /$ ncomms4596)

8 Ma W, Zhang CQ, Dang CX, Cai HY, Li HL, Miao GY, Wang JK \& Zhang LJ. Upregulated long-non-coding RNA DLEU2 exon 9 expression was an independent indicator of unfavorable overall survival in patients with esophageal adenocarcinoma. Biomedicine and Pharmacotherapy 2019113 108655. (https://doi.org/10.1016/j. biopha.2019.108655)

9 Xie ZZ, Xiao ZC, Song YX, Li W \& Tan GL. Long non-coding RNA Dleu2 affects proliferation, migration and invasion ability of laryngeal carcinoma cells through triggering miR-16-1 pathway. European Review for Medical and Pharmacological Sciences 201822 1963-1970. (https://doi.org/10.26355/eurrev_201804_14723)

10 Liu H, Zhang Z, Wu N, Guo H, Zhang H, Fan D, Nie Y \& Liu Y. Integrative analysis of dysregulated IncRNA-associated ceRNA network reveals functional lncRNAs in gastric cancer. Genes 20189 303. (https://doi.org/10.3390/genes9060303)
11 Xie Z, Li X, Chen H, Zeng A, Shi Y \& Tang Y. The IncRNA-DLEU2/ miR-186-5p/PDK3 axis promotes the progress of glioma cells. American Journal of Translational Research 201911 4922-4934.

12 Zhang H, Cai Y, Zheng L, Zhang Z, Lin X \& Jiang N. Long noncoding RNA NEAT1 regulate papillary thyroid cancer progression by modulating miR-129-5p/KLK7 expression. Journal of Cellular Physiology 2018233 6638-6648. (https://doi.org/10.1002/ jср.26425)

13 Croce CM \& Calin GA. miRNAs, cancer, and stem cell division. Cell 2005122 6-7. (https://doi.org/10.1016/j.cell.2005.06.036)

14 Ambros V. The functions of animal microRNAs. Nature $2004 \mathbf{4 3 1}$ 350-355. (https://doi.org/10.1038/nature02871)

15 Calin GA \& Croce CM. MicroRNA signatures in human cancers. Nature Reviews: Cancer 20066 857-866. (https://doi.org/10.1038/ nrc1997)

16 Bartel DP. MicroRNAs: genomics, biogenesis, mechanism, and function. Cell 2004116 281-297. (https://doi.org/10.1016/s00928674(04)00045-5)

17 Vosgha H, Salajegheh A, Smith RA \& Lam AK. The important roles of miR-205 in normal physiology, cancers and as a potential therapeutic target. Current Cancer Drug Targets 201414 621-637. (https://doi.org/ $10.2174 / 156800961407140926105634)$

18 Li L \& Li S. miR-205-5p inhibits cell migration and invasion in prostatic carcinoma by targeting ZEB1. Oncology Letters $2018 \mathbf{1 6}$ 1715-1721. (https://doi.org/10.3892/ol.2018.8862)

19 Tao W, Sun W, Zhu H \& Zhang J. miR-205-5p suppresses pulmonary vascular smooth muscle cell proliferation by targeting MICAL2mediated ERK1/2 signaling. Microvascular Research 2019124 43-50. (https://doi.org/10.1016/j.mvr.2019.03.001)

20 Vosgha H, Ariana A, Smith RA \& Lam AK. miR-205 targets angiogenesis and EMT concurrently in anaplastic thyroid carcinoma. Endocrine-Related Cancer 201825 323-337. (https://doi.org/10.1530/ ERC-17-0497)

21 Niture S, Dong X, Arthur E, Chimeh U, Niture SS, Zheng W \& Kumar D. Oncogenic role of tumor necrosis factor alpha-induced protein 8 (TNFAIP8). Cells 2018 8. (https://doi.org/10.3390/ cells8010009)

22 Xing Y, Liu Y, Liu T, Meng Q, Lu H, Liu W, Hu J, Li C, Cao M, Yan $\mathrm{S}$, et al. TNFAIP8 promotes the proliferation and cisplatin chemoresistance of non-small cell lung cancer through MDM2/p53 pathway. Cell Communication and Signaling 201816 43. (https://doi. org/10.1186/s12964-018-0254-x)

23 Niture S, Ramalinga M, Kedir H, Patacsil D, Niture SS, Li J, Mani H, Suy S, Collins S \& Kumar D. TNFAIP8 promotes prostate cancer cell survival by inducing autophagy. Oncotarget $2018926884-26899$. (https://doi.org/10.18632/oncotarget.25529)

24 Xiao M, Xu Q, Lou C, Qin Y, Ning X, Liu T, Zhao X, Jia S \& Huang Y. Overexpression of TNFAIP8 is associated with tumor aggressiveness and poor prognosis in patients with invasive ductal breast carcinoma. Human Pathology 201762 40-49. (https://doi. org/10.1016/j.humpath.2016.12.020)

25 Duan D, Zhu YQ, Guan LL \& Wang J. Upregulation of SCC-S2 in immune cells and tumor tissues of papillary thyroid carcinoma. Tumour Biology 201435 4331-4337. (https://doi.org/10.1007/ s13277-013-1568-3)

26 Livak KJ \& Schmittgen TD. Analysis of relative gene expression data using real-time quantitative PCR and the 2(-Delta Delta C(T)) Method. Methods 200125 402-408. (https://doi.org/10.1006/ meth.2001.1262)

27 Zhao SJ, Shen YF, Li Q, He YJ, Zhang YK, Hu LP, Jiang YQ, Xu NW, Wang YJ, Li J, et al. SLIT2/ROBO1 axis contributes to the Warburg effect in osteosarcoma through activation of SRC/ERK/c-MYC/ PFKFB2 pathway. Cell Death and Disease 20189 390. (https://doi. org/10.1038/s41419-018-0419-y) https://ec.bioscientifica.com https://doi.org/10.1530/EC-21-0046 (c) 2021 The authors Published by Bioscientifica Ltd

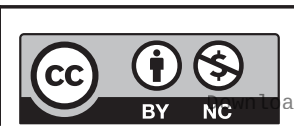

This work is licensed under a Creative Commons Attribution-NonCommercial 4.0 International License. ded from Bioscientifica.com at $04 / 26 / 2023$ 12:36:34PM 
28 Koppenol WH, Bounds PL \& Dang CV. Otto Warburg's contributions to current concepts of cancer metabolism. Nature Reviews: Cancer 201111 325-337. (https://doi.org/10.1038/nrc3038)

29 Zheng X, Boyer L, Jin M, Mertens J, Kim Y, Ma L, Ma L, Hamm M, Gage FH \& Hunter T. Metabolic reprogramming during neuronal differentiation from aerobic glycolysis to neuronal oxidative phosphorylation. eLife 20165 e13374. (https://doi.org/10.7554/ eLife.13374)

30 Guo Y, Bai M, Lin L, Huang J, An Y, Liang L, Liu Y \& Huang W. lncRNA DLEU2 aggravates the progression of hepatocellular carcinoma through binding to EZH2. Biomedicine and
Pharmacotherapy 2019118 109272. (https://doi.org/10.1016/j. biopha.2019.109272)

31 Xu B, Gong X, Zi L, Li G, Dong S, Chen X \& Li Y. Silencing of DLEU2 suppresses pancreatic cancer cell proliferation and invasion by upregulating microRNA-455. Cancer Science 2019110 1676-1685. (https://doi.org/10.1111/cas.13987)

32 Zhou Y, Shi H, Du Y, Zhao G, Wang X, Li Q, Liu J, Ye L, Shen Z, Guo Y, et al. IncRNA DLEU2 modulates cell proliferation and invasion of non-small cell lung cancer by regulating miR-30c-5p/ SOX9 axis. Aging 201911 7386-7401. (https://doi.org/10.18632/ aging.102226)

Received in final form 2 March 2021

Accepted 24 March 2021

Accepted Manuscript published online 25 March 2021
This work is licensed under a Creative Commons Attribution-NonCommercial 4.0 International License. 